\title{
SOLVABLE NORMAL SUBGROUPS OF 2-KNOT GROUPS
}

\author{
J.A.HILLMAN
}

\begin{abstract}
If $X$ is an orientable, strongly minimal $P D_{4}$-complex and $\pi_{1}(X)$ has one end then it has no nontrivial locally-finite normal subgroup. Hence if $\pi$ is a 2-knot group then (a) if $\pi$ is virtually solvable then either $\pi$ has two ends or $\pi \cong \Phi$, with presentation $\left\langle a, t \mid t a=a^{2} t\right\rangle$, or $\pi$ is torsion-free and polycyclic of Hirsch length 4 ; (b) either $\pi$ has two ends, or $\pi$ has one end and the centre $\zeta \pi$ is torsion-free, or $\pi$ has infinitely many ends and $\zeta \pi$ is finite; and (c) the Hirsch-Plotkin radical $\sqrt{\pi}$ is nilpotent.
\end{abstract}

A 2-knot is a topologically locally flat embedding of $S^{2}$ in $S^{4}$. The main result of this note is that if the group of a 2-knot is virtually solvable then it either has finite commutator subgroup or is torsionfree. All such groups are known, and in the torsion-free case the knot exteriors are determined up to homeomorphism by the knot group $\pi$ together with the $A u t(\pi)$-orbit of a meridian. With the exception of one group, such knots may be constructed by elementary surgery on a section of the mapping torus of a diffeomorphism of a 3-manifold; the cocore of the surgery is then a smooth 2-knot in a smooth homotopy 4 -sphere. (The exceptional group is the group $\Phi$ of Examples 10 and 11 in [5], which are ribbon knots.) In many cases the homotopy 4-sphere is standard, but the question remains open in general. (This construction is perhaps the simplest source for possible counter-examples to the smooth 4-dimensional Poincaré conjecture [8].)

The first section establishes some notation. In $\S 2$ we show that if $X$ is an orientable $P D_{4}$-complex such that $\pi_{1}(X)$ has one end and the equivariant intersection pairing on $\pi_{2}(X)$ is 0 then $\pi_{1}(X)$ has no nontrivial locally-finite normal subgroup. This is used in $\S 3$ to show that if a 2-knot group $\pi$ is virtually solvable then either $\pi^{\prime}$ is finite or $\pi \cong \Phi=Z *_{2}$, with presentation $\left\langle a, t \mid t a=a^{2} t\right\rangle$, or $\pi$ is torsion-free polycyclic and of Hirsch length 4. (The final family of such groups was found in [14.) More generally, if $S$ is an infinite solvable normal

1991 Mathematics Subject Classification. 57Q45.

Key words and phrases. centre, coherent, Hirsch-Plotkin radical, 2-knot, torsion. 
subgroup and $\pi$ is not itself solvable then $S \cong \mathbb{Z}^{2}$ or is virtually torsionfree abelian of rank 1 . We show also that the Hirsch-Plotkin radical $\sqrt{\pi}$ of every 2-knot group is nilpotent. Finally we consider the centre $\zeta \pi$. If $\pi$ has one end then $\zeta \pi \cong \mathbb{Z}^{2}$ or is torsion-free, of rank $\leq 1$. In particular, this is so if the commutator subgroup $\pi^{\prime}$ is infinite and $\zeta \pi$ has rank $>0$. If $\pi$ has two ends $\zeta \pi$ has rank 1 , and may be either $\mathbb{Z}$ or $\mathbb{Z} \oplus Z / 2 Z$, while if $\pi$ has infinitely many ends $\zeta \pi$ is finite. We extend a construction of [27] to give examples with $\sqrt{\pi}$ cyclic of order $q$ or $2 q$, with $q$ odd.

The group $\Phi$ is the only nontrivial higher dimensional knot group which is solvable and has deficiency 1 . In $\S 4$ we sketch briefly how the determination of 2-knots with this group might extend to other groups with geometric dimension 2 , and perhaps to all with deficiency 1 . In view of the present limitations on 4-dimensional surgery techniques, we can expect only a classification up to $s$-concordance (relative $s$ cobordism of knots). With this proviso, we can show that a 2 -knot whose group $\pi$ is a 1-knot group is determined up to $s$-concordance, reflections and the Gluck ambiguity (whether a knot is determined by its exterior [7]) by $\pi$ and the $A u t(\pi)$-orbit of a meridian (Theorem 5). In particular, a 2-knot with group $\pi$ and meridian $\mu$ is $s$-concordant to the Artin spin of a fibred 1-knot if and only if $\pi^{\prime}$ is finitely generated, $C_{\pi}(\mu) \cong \mathbb{Z}^{2}$ and $\left(\pi, C_{\pi}(\mu)\right)$ is a $P D_{3}$-pair (Corollary 6.1 ).

\section{NOTATION}

If $G$ is a group let $\zeta G$ and $G^{\prime}$ be the centre and commutator subgroup of $G$, respectively. Let $C_{G}(H)$ be the centralizer of a subgroup $H \leq G$. The product of locally-nilpotent normal subgroups of $G$ is again a locally-nilpotent normal subgroup, by the Hirsch-Plotkin Theorem, and the Hirsch-Plotkin radical $\sqrt{G}$ is the (unique) maximal such subgroup. (See Proposition 12.1.2 of [23].) In general, $\sqrt{G}$ is elementary amenable, but need not be nilpotent.

If $E$ is an elementary amenable group then it has a well-defined Hirsch length $h(E) \in \mathbb{N} \cup\{\infty\}$. (See [15] or Chapter 1 of [11].)

If $X$ is a space let $\beta_{i}^{(2)}(X)$ be the $i$ th $L^{2}$ Betti number of $X$, and let $\beta_{i}^{(2)}(G)=\beta_{i}^{(2)}(K(G, 1))$. (See [21] for a comprehensive exposition of $L^{2}$-theory.)

\section{2. $P D_{4}$-COMPLEXES WiTH $\chi=0$}

A $P D_{4}$-complex $X$ with fundamental group $\pi$ is strongly minimal if the equivariant intersection pairing on $\pi_{2}(X)$ is 0 , equivalently, if the homomorphism from $H^{2}(\pi ; \mathbb{Z}[\pi])$ to $H^{2}(X ; \mathbb{Z}[\pi])$ induced by the 
classifying map $c_{X}: X \rightarrow K(\pi, 1)$ is an isomorphism [12]. Note that $X$ is aspherical if and only if it is strongly minimal and $H^{i}(\pi ; \mathbb{Z}[\pi])=0$ for $i \leq 2$.

Lemma 1. Let $X$ be a finite $P D_{4}$-complex with fundamental group $\pi$. If $\chi(X)=0$ and $\beta_{1}^{(2)}(\pi)=0$ then $X$ is strongly minimal.

Proof. Since $X$ is a finite complex the $L^{2}$-Euler characteristic formula holds, and so $\chi(X)=\beta_{2}^{(2)}(X)-2 \beta_{1}^{(2)}(X)$. Hence $\beta_{2}^{(2)}(X)=0$ also. Since $\beta_{1}^{(2)}(X)=\beta_{1}^{(2)}(\pi)=0, \beta_{2}^{(2)}(X) \geq \beta_{2}^{(2)}(\pi) \geq 0$ and $\chi(X)=0$, it follows that $\beta_{2}^{(2)}(X)=\beta_{2}^{(2)}(\pi)$. Hence $H^{2}\left(c_{X} ; \mathbb{Z}[\pi]\right)$ is an isomorphism, by part (3) of Theorem 3.4 of [11].

The condition $\beta_{1}^{(2)}(\pi)=0$ holds if $\pi$ has an infinite normal subgroup which is amenable, or is finitely generated and of infinite index. (See Chapter 7 of [21].)

Lemma 2. Let $G$ be a group. If $T$ is a locally finite normal subgroup of $G$ then $T$ acts trivially on $H^{j}(G ; \mathbb{Z}[G])$, for all $j \geq 0$.

Proof. If $T$ is finite then $\left.H^{j}(G ; \mathbb{Z}[G]) \cong H^{j}(G / T ; \mathbb{Z}[G / T])\right)$, for all $j$, and the result is clear. Thus we may assume that $T$ and $G$ are infinite. Hence $H^{0}(G ; \mathbb{Z}[G])=0$, and $T$ acts trivially. We may write $T=\cup_{n \geq 1} T_{n}$ as a strictly increasing union of finite subgroups. Then there are short exact sequences [16]

$$
0 \rightarrow \lim ^{1} H^{s-1}\left(T_{n} ; \mathbb{Z}[\pi]\right) \rightarrow H^{s}(T ; \mathbb{Z}[\pi]) \rightarrow \underset{\longleftarrow}{\lim } H^{s}\left(T_{n} ; \mathbb{Z}[\pi]\right) \rightarrow 0 .
$$

Hence $H^{s}(T ; \mathbb{Z}[\pi])=0$ if $s \neq 1$ and $H^{1}(T ; \mathbb{Z}[\pi])=\lim ^{1} H^{0}\left(T_{n} ; \mathbb{Z}[\pi]\right)$, and so the LHS spectral sequence collapses to give $H^{j}(G ; \mathbb{Z}[G]) \cong$ $H^{j-1}\left(G / T ; H^{1}(T ; \mathbb{Z}[G])\right)$, for all $j \geq 1$. Let $g \in T$. We may assume that $g \in T_{n}$ for all $n$, and so $g$ acts trivially on $H^{0}\left(T_{n} ; \mathbb{Z} G\right)$, for all $j$ and $n$. But then $g$ acts trivially on $\lim ^{1} H^{0}\left(T_{n} ; \mathbb{Z}[\pi]\right)$, by the functoriality of the construction. Hence every element of $T$ acts trivially on $H^{j-1}\left(G / T ; H^{1}(T ; \mathbb{Z}[G])\right)$, for all $j \geq 1$.

If $\pi$ is a group and $M$ is a right $\mathbb{Z}[\pi]$-module let $\bar{M}$ be the conjugate left module, with $\pi$-action defined by $g . m=m g^{-1}$ for all $m \in M$ and $g \in \pi$.

Theorem 3. Let $X$ be an orientable $P D_{4}$-complex with fundamental group $\pi$. If $X$ is strongly minimal and $\pi$ has one end then $\pi$ has no non-trivial locally-finite normal subgroup.

Proof. Let $\widetilde{X}$ be the universal cover of $X$, and let $C_{*}=C_{*}(X ; \mathbb{Z}[\pi])=$ $C_{*}(\widetilde{X} ; \mathbb{Z})$ be the cellular chain complex of $\tilde{X}$. We assume that $\pi$ acts 
on the left on $\tilde{X}$, and so $C_{*}$ is a complex of free left $\mathbb{Z}[\pi]$-modules. Since $\pi$ has one end, $H_{s}(\widetilde{X} ; \mathbb{Z})=H_{s}(X ; \mathbb{Z}[\pi])=0$ for $s \neq 0$ or 2 . Let $\Pi=\pi_{2}(X) \cong H_{2}(\widetilde{X} ; \mathbb{Z})=H_{2}(X ; \mathbb{Z}[\pi])$. Poincaré duality and $c_{X}$ give an isomorphism $\Pi \cong \overline{H^{2}(X ; \mathbb{Z}[\pi])}$. Since $X$ is strongly minimal, this in turn is isomorphic to $\overline{H^{2}(\pi ; \mathbb{Z}[\pi])}$.

Suppose that $\pi$ has a nontrivial locally-finite normal subgroup $T$. Let $g \in T$ have prime order $p$, and let $C=\langle g\rangle \cong Z / p Z$. Then $C$ acts freely on $\widetilde{X}$, which has homology only in degrees 0 and 2 . On considering the homology spectral sequence for the classifying map $c_{\tilde{X} / C}: \widetilde{X} / C \rightarrow K(C, 1)$, we see that $H_{i+3}(C ; \mathbb{Z}) \cong H_{i}(C ; \Pi)$, for all $i \geq 2$. (See Lemma 2.10 of [11].) Since $C$ has cohomological period 2 and acts trivially on $\Pi$, by Lemma 1 , there is an exact sequence

$$
0 \rightarrow Z / p Z \rightarrow \Pi \rightarrow \Pi \rightarrow 0 \text {. }
$$

On the other hand, since $\pi$ is finitely presentable, $\Pi \cong H^{2}(\pi ; \mathbb{Z}[\pi])$ is torsion-free, by Proposition 13.7.1 of [6]. Hence $T$ has no such element $g$ and so $\pi$ has no such finite normal subgroup.

Corollary 3.1. Every ascending locally finite subgroup of $\pi$ is trivial.

Proof. If $T$ is an ascending locally finite subgroup of $\pi$ then a transfinite induction shows that the normal closure of $T$ in $\pi$ is locally finite.

Theorems 8.1 and 9.1 of [11] are the bases for characterizations of the homotopy types of 4-dimensional infrasolvmanifolds and Seifert fibred 4-manifolds, respectively. Each assumes at some point that $H^{2}(\pi ; \mathbb{Z}[\pi])=0$. We have long felt that this condition might be redundant. The results above justify this expectation for Theorem 9.1, which may now be stated as follows.

Theorem. Let $M$ be a closed 4-manifold with fundamental group $\pi$. If $\chi(M)=0$ and $\pi$ has an elementary amenable normal subgroup $\rho$ with $h(\rho)=2$ and $[\pi: \rho]=\infty$ then $M$ is aspherical and $\rho$ is virtually abelian.

The key points are that $\pi$ has one end, since $h(\rho)>1$, and the quotient of $\rho$ by its maximal locally-finite normal subgroup $\tau$ is virtually solvable, since $h(\rho)<\infty$. Hence $\tau=1$, so $\rho$ is virtually solvable, and then $\pi$ has a nontrivial torsion-free abelian normal subgroup. Theorem 1.17 of [11] may then be used to show that $H^{2}(\pi ; \mathbb{Z}[\pi])=0$, and the rest of the argument is as in [11].

We do not yet have a corresponding improvement of Theorem 8.1, as we do not know how to show that $H^{2}(\rho ; W)=0$ if $\rho$ is elementary 
amenable, has no nontrivial locally-finite normal subgroup and $h(\rho)=$ $\infty$, and $W$ is a free $\mathbb{Z}[\rho]$-module. (This holds if $\rho$ is torsion-free $[20]$.)

\section{CENTRES, HIRSCH-PLOTKIN RADICALS AND VIRTUALLY SOLVABLE 2-KNOT GROUPS}

We work with topological manifolds and locally flat embeddings. Thus a 2-knot is an embedding $K: S^{2} \rightarrow S^{4}$ which extends to a product neighbourhood $N(K) \cong S^{2} \times D^{2}$. The knot exterior is $X(K)=$ $\overline{S^{4} \backslash N(K)}$, and the knot group is $\pi=\pi K=\pi_{1}(X(K))$. Let $M(K)=$ $X(K) \cup D^{3} \times S^{1}$ be the closed 4-manifold obtained by elementary surgery on $K$. Then $\pi_{1}(M(K)) \cong \pi$ and $\chi(M(K))=0$.

We can recover the exterior from the knot manifold $M(K)$ by specifying the isotopy class of the core $\{0\} \times S^{1}$. By general position, this is determined by the conjugacy class of a meridian. A weight orbit in a knot group $\pi$ is the orbit of a normal generator under the action of $A u t(\pi)$. If each automorphism of $\pi$ is realizable by a self-homeomorphism of $M(K)$ then $X(K)$ is determined by $M(K)$ together with the weight orbit of a meridian for $K$. (In general, a 2-knot group may have many distinct weight orbits, corresponding to distinct knots giving rise to the same knot manifold. See $\S 7$ of Chapter 14 of [11].)

If we reattach $S^{2} \times D^{2}$ to $X(K)$ via an identification $\partial X(K) \cong$ $S^{2} \times S^{1}$ we may recover a 2-knot, represented by the core $S^{2} \times\{0\}$. However there is a potential ambiguity of order 2 here, since $S^{n} \times S^{1}$ has an involution which does not extend across $S^{n} \times D^{2}$, when $n>1$ [7]. If this involution extends across $X(K)$ then $K$ is determined by its exterior; we say that $K$ is reflexive.

Theorem 4. Let $K$ be a 2-knot with group $\pi=\pi K$. If $\pi$ has normal subgroups $A \leq E$ with $A$ a nontrivial abelian group and $E$ an infinite elementary amenable group then either $\pi^{\prime}$ is finite or $E$ is virtually torsion-free solvable. If $h(E)=1$ then $E$ is abelian or virtually $\mathbb{Z}$; if $h(E)=2$ then $E \cong \mathbb{Z}^{2}$, and if $h(E)>2$ then $E$ is torsion-free polycyclic, and $h(E)=3$ or 4 .

Proof. We may assume that $\pi^{\prime}$ is infinite. Then $\pi$ has one end, and $\beta_{1}^{(2)}(\pi)=0$, since $\pi$ has an infinite amenable normal subgroup. Since $M(K)$ is a closed 4-manifold, it is homotopy equivalent to a finite $P D_{4}$-complex, and so is strongly minimal, by Lemma 1 . The torsion subgroup of $A$ is characteristic, and so is normal in $\pi$. Hence $A$ is torsion-free, by Theorem 3. Therefore either $\pi \cong \Phi$ or $M(K)$ is aspherical or $A \cong \mathbb{Z}$ and $\pi / A$ has infinitely many ends, by Theorem 15.7 
of [11]. If $M(K)$ is aspherical then $E$ has finite cohomological dimension, and so is virtually solvable [15]. (See Corollary 1.9.2 of [11].) If $\pi / A$ has infinitely many ends then $E / A$ is finite, since it is an elementary amenable normal subgroup in $\pi / A$. In all cases $E$ is in fact virtually torsion-free solvable.

Since $\pi$ has one end, $E$ has no non-trivial finite normal subgroup. Hence if $h(E)=1$ then $\sqrt{E}$ is torsion-free, abelian and of index $\leq 2$ in $E$. If $E$ is not finitely generated, neither is $\sqrt{E}$. In this case $\pi \cong \Phi$ or $M(K)$ is aspherical (by Theorem 15.7 of [11], as above), so $\pi$ is torsion-free, and $E$ must be abelian.

If $h(E)=2$ then $M(K)$ is aspherical, $E$ is torsion-free and $\pi / E$ is virtually a $P D_{2}$-group, by Theorems 9.1 and 16.2 of [11]. Since $M(K)$ is orientable, $E$ cannot be the Klein bottle group, and so $E \cong \mathbb{Z}^{2}$. If $h(E)>2$ then $\pi$ is torsion-free polycyclic and $h(\pi)=4$, by Theorem 8.1 of [1], so $h(E)=3$ or 4 .

Corollary 4.1. The following are equivalent:

(1) $\pi$ is elementary amenable and $h(\pi)<\infty$;

(2) $\pi$ is virtually solvable;

(3) either $\pi^{\prime}$ is finite or $\pi \cong \Phi$ or $\pi$ is torsion-free polycyclic and $h(\pi)=4$.

Proof. If $\pi$ is elementary amenable and $h(\pi)<\infty$ then either $\pi^{\prime}$ is finite or $\pi$ has one end. In the latter case $\pi$ has no nontrivial locally-finite normal subgroup, by Lemma 1 and Theorem 3, and so $\pi$ is virtually solvable, by Corollary 1.9.2 of [11].

If $\pi$ has a solvable normal subgroup $S$ of finite index then the lowest nontrivial term of the derived series for $S$ is abelian, and is characteristic in $S$. Hence it is normal in $\pi$, and Theorem 4 applies.

Finally, (3) clearly implies the other conditions.

It is enough to assume that $\pi$ is elementary amenable and has a nontrivial abelian normal subgroup. Can we relax "virtually solvable" further to "elementary amenable", or even just "amenable"?

If $\pi \cong \Phi$ then $K$ is TOP isotopic to Fox's Example 10 or its reflection Example 11 [12, while if $\pi$ is torsion-free polycyclic then it is one of the groups described in $\S 4$ of Chapter 16 of [11], and $\pi$ determines $M(K)$ up to homeomorphism, by Theorem 17.4 of [11].

Corollary 4.2. The Hirsch-Plotkin radical $\sqrt{\pi}$ is nilpotent.

Proof. Since $\sqrt{\pi}$ is locally nilpotent it has a maximal locally-finite normal subgroup $T$ with torsion-free quotient. If $\sqrt{\pi}$ is finitely generated there is nothing to prove. Otherwise, $\pi$ has one end and so $T$ is trivial, by Theorem 3. If $T=1$ and $h(\sqrt{\pi}) \leq 2$ then $\sqrt{\pi}$ is abelian; if 
$h(\sqrt{\pi})>2$ then $\pi$ is virtually polycyclic and $h(\pi)=4$, by Theorem 8.1 of [11]. In each case $\sqrt{\pi}$ is nilpotent.

Every finitely generated abelian group is the centre of some highdimensional knot group [10]. On the other hand, the only 1-knots whose groups have nontrivial abelian normal subgroups are the torus knots, for which $\sqrt{\pi}=\zeta \pi \cong \mathbb{Z}$ and $\zeta \pi \cap \pi^{\prime}=1$. The intermediate case of 2 -knots is less clear. If $\zeta \pi$ has rank $>1$ then it is $\mathbb{Z}^{2}$; most twist spins of torus knots have such groups. There are examples with centre $1, Z / 2 Z, \mathbb{Z}$ or $\mathbb{Z} \oplus Z / 2 Z$. (See Chapters $15-17$ of [11].)

Corollary 4.3. Let $K$ be a 2-knot with group $\pi=\pi K$. Then

(1) if $\pi$ has two ends then $\zeta \pi \cong \mathbb{Z} \oplus Z / 2 Z$ if $\pi^{\prime}$ has even order; otherwise $\zeta \pi \cong \mathbb{Z}$;

(2) if $\pi$ has one end then $\zeta \pi \cong \mathbb{Z}^{2}$, or is torsion-free of rank $\leq 1$;

(3) if $\pi$ has infinitely many ends then $\zeta \pi$ is finite.

Proof. If $\pi$ has two ends then $\pi^{\prime}$ is finite, and so $\zeta \pi$ is finitely generated and of rank 1 . It follows from the classification of such 2-knot groups (see Chapter 15 of [11]) that $\zeta \pi \cong \mathbb{Z} \oplus Z / 2 Z$ if $\pi^{\prime}$ has even order, and otherwise $\zeta \pi \cong \mathbb{Z}$.

Part (2) follows from Theorem 4, while part (3) is clear.

Note that $\pi$ has finitely many ends if $\pi^{\prime}$ is finitely generated, or if $\zeta \pi$ is infinite. When $\pi$ has more than one end Lemma 2.10 of [11] either does not lead to a contradiction or does not apply.

If $\zeta \pi$ is a nontrivial torsion group then it is finite. Yoshikawa constructed an example of a 2-knot whose group $\pi$ has centre of order 2 [28. It is easy to see that $\sqrt{\pi}=\zeta \pi$ in this case. The construction may be extended as follows. Let $q>0$ be odd and let $k_{q}$ be a 2-bridge knot such that the 2-fold branched cyclic cover of $S^{3}$, branched over $k_{q}$ is a lens space $L(3 q, r)$, for some $r$ relatively prime to $q$. Let $K_{1}=\tau_{2} k_{q}$ be the 2-twist spin of $k_{q}$, and let $K_{2}=\tau_{3} k$ be the 3 -twist spin of a nontrivial knot $k$. Let $\gamma$ be a simple closed curve in $X\left(K_{1}\right)$ with image $[\gamma] \in \pi K_{1}$ of order $3 q$, and let $w$ be a meridian for $K_{2}$. Then $w^{3}$ is central in $\pi K_{2}$. The group of the satellite of $K_{1}$ about $K_{2}$ relative to $\gamma$ is the generalized free product

$$
\pi=\pi K_{2} /\left\langle\left\langle w^{3 q}\right\rangle\right\rangle *_{w=[\gamma]} \pi K_{1} .
$$

(See [17].) Hence $\sqrt{\pi}=\left\langle w^{3}\right\rangle \cong Z / q Z$, while $\zeta \pi=1$.

If we use a 2 -knot $K_{1}$ with $\operatorname{group}(Q(8) \times Z / 3 q Z) \rtimes_{\theta} \mathbb{Z}$ instead and choose $\gamma$ so that $[\gamma]$ has order $6 q$ then we obtain examples with $\sqrt{\pi} \cong Z / 2 q Z$ and $\zeta \pi=Z / 2 Z$. (Knots $K_{1}$ with such groups may be 
constructed by surgery on sections of mapping tori of homeomorphisms of 3-manifolds with fundamental group $Q(8) \times Z / 3 q Z$ [27].)

If $\zeta \pi$ has rank 1 and nontrivial torsion then $\pi^{\prime}$ is finite, and $\zeta \pi$ is finitely generated.

If $\zeta \pi$ has rank 1 but is not finitely generated then $M(K)$ is aspherical. It is not known whether there are such 2-knots (nor, more generally, whether abelian normal subgroups of rank 1 in $P D_{n}$-groups with $n>3$ must be finitely generated). What little we know about this case is as follows. Since $\zeta \pi<\pi^{\prime}$ and $\pi / \pi^{\prime} \cong \mathbb{Z}$, we must have $\zeta \pi \leq \pi^{\prime \prime}$. Since $\zeta \pi$ is torsion-free of rank 1 but is not finitely generated, c.d. $\zeta \pi=2$. Hence if $G$ is a nonabelian subgroup which contains $\zeta \pi$ then c.d.G $\geq 3$, by Theorem 8.6 of [2]. If $H$ is a subgroup of $\pi$ such that $H \cap \zeta \pi=1$ then $H . \zeta \pi \cong H \times \zeta \pi$ is not finitely generated, and so has infinite index in $\pi$. Hence $c . d . H \times \mathbb{Z} \leq$ c.d. $H \times \zeta \pi \leq 3$ [24]. Theorem 5.5 of [2] gives, firstly, that c.d. $H \leq 2$, and then, that if $H$ is $F P_{2}$ then c.d. $H \leq 1$, and so $H$ is free. Thus if $\pi$ is almost coherent every subgroup either meets $\zeta \pi$ nontrivially or is locally free.

If $\zeta \pi$ has rank $>1$ then $M(K)$ is aspherical and $\zeta \pi \cong \mathbb{Z}^{2}$, by Theorem 16.3 of [11].

The following questions remain open:

(i) if $\zeta \pi$ has rank 1 , must it be finitely generated?

(ii) if $\zeta \pi$ is finite, must it be $Z / 2 Z$ or 1 ?

(iii) is there a 2 -knot group $\pi$ with $\sqrt{\pi}$ a non-cyclic finite group?

(iv) if $\pi$ is elementary amenable is it virtually solvable?

In each case the answer is "yes" if $\pi^{\prime}$ is finitely presentable, for then the infinite cyclic cover $M(K)^{\prime}$ is homotopy equivalent to a $P D_{3}$-complex, by Theorem 4.5 of [11.

\section{GEOMETRIC DIMENSION 2}

The only solvable 2-knot groups with deficiency 1 are $\mathbb{Z}$ and $\Phi$. One of the early triumphs of 4-dimensional surgery was Freedman's unknotting criterion: 2 -knots with group $\mathbb{Z}$ are topologically trivial. In 12 we showed that any 2 -knot $K$ with group $\Phi$ is topologically equivalent to Fox's Example 10 or its reflection (Example 11). The argument involved showing that

(i) $\pi=\pi K$ determines the homotopy type of $M(K)$;

(ii) $W h(\pi)=0$ and the surgery assembly homomorphisms $\mathcal{A}_{*}(\pi)$ : $H_{*}\left(\pi ; \mathbb{L}_{0}\right) \rightarrow L_{*}(\pi)$ are isomorphisms;

(iii) 4-dimensional TOP surgery;

(iv) $\pi$ has an unique weight orbit, up to inversion; and

(v) Fox's examples are reflexive. 
The group $\Phi$ has geometric dimension $2($ g.d. $\Phi=2)$ : there is a finite 2-dimensional $K(\Phi, 1)$ complex. Our strategy for (i) uses the fact that $M(K)$ is strongly minimal if $g . d . \pi=2$, since $\chi(M(K))=0=2 \chi(\pi)$. It should extend to all knot groups $\pi$ with $g . d . \pi=2$, but at present is incomplete; there is a 2-torsion condition which holds for $\Phi$, but is not otherwise easily verified. (See [13] for a much expanded exposition of [12] and earlier work.) When g.d. $\pi=2$ and $\pi^{\prime}$ is finitely generated there is a quite different argument. For then $\pi^{\prime}$ is free of finite rank, and $M(K)$ is homotopy equivalent to the mapping torus of a self-homeomorphism of $\#^{r}\left(S^{2} \times S^{1}\right)$, where $r$ is the rank of $\pi^{\prime}$. (See Corollary 4.5.3 of [11].)

Step (ii) holds if $\pi$ is a 1-knot group, or is square root closed accessible, or is in the class $\mathcal{X}$ of fundamental groups of finite graphs of groups with all vertex and edge groups $\mathbb{Z}$. (See Lemma 6.9 of [11.) Semidirect products $F(r) \rtimes \mathbb{Z}$ are square root closed accessible, while $\Phi$ is in $\mathcal{X}$.

At the time of writing, the largest known class of groups for which 4-dimensional topological surgery works is the class $S G$ obtained from subexponential groups by taking increasing unions and extensions. To go beyond this class we must work modulo TOP $s$-cobordisms (or $s$ concordances of 2-knots) rather than expect homeomorphisms.

Step (iv) fails for other knot groups $\pi$ with $g . d . \pi=2$. In general, we must specify a weight orbit in order to recover $X(K)$ from $M(K)$.

The final step (v) is not satisfied by all 2-knots. However, those which have a Seifert hypersurface with free fundamental group are reflexive. Fibred 2-knots with $\pi^{\prime}$ free and ribbon 2-knots have such Seifert hypersurfaces [26].

The difficulty with (i) has been bypassed in 9, which shows directly that an orientable 4-manifold $M$ with $\pi=\pi_{1}(M)$ such that $g . d . \pi=2$ and $\pi$ "satisfies properties $W$-AA" (i.e., (ii) holds) is determined up to $s$-cobordism by $\pi$, the second $\mathrm{Wu}$ class $w_{2}(M)$ and the equivariant intersection pairing on $\pi_{2}(M)$.

Theorem 5. Let $\pi$ be a 2-knot group with deficiency 1 . If either

(1) $\pi$ is the group of a 1-knot; or

(2) $\pi^{\prime}$ is finitely generated; or

(3) $\beta_{1}^{(2)}(\pi)=0$ and $\pi$ is square-root closed accessible,

then any 2-knot with group $\pi$ is determined up to TOP s-concordance, reflections and the Gluck ambiguity by $\pi$ and the weight orbit of a meridian.

Proof. We may assume that $\pi \approx \mathbb{Z}$. 
If $\pi$ is a 1-knot group then $g . d . \pi=2$ and $\beta_{1}^{(2)}(\pi)=0$, by Theorem 4.1 of [21], while $W h(\pi)=0$ and the surgery assembly homomorphisms $\mathcal{A}_{*}(\pi)$ are isomorphisms [1]. If $\pi^{\prime}$ is finitely generated then $\beta_{1}^{(2)}(\pi)=0$, while if $\operatorname{def}(\pi)=1$ and $\beta_{1}^{(2)}(\pi)=0$ then $g . d . \pi=2$, by Theorem 2.5 of [11. In particular, if $\pi^{\prime}$ is finitely generated and $\operatorname{def}(\pi)=1$ then $\pi^{\prime}$ is free and so $\pi \cong \pi^{\prime} \rtimes \mathbb{Z}$ is square root closed accessible. Hence $(2) \Rightarrow(3)$, which implies that $W h(\pi)=0$ and the $\mathcal{A}_{*}(\pi)$ are isomorphisms [3]. Thus in each case $\pi$ satisfies properties $W-A A$ of [9].

In each case $\pi$ is torsion-free, and so has one end [19], and $\beta_{1}^{(2)}(\pi)=$ 0 . Hence $M(K)$ is strongly minimal, by Lemma 1 . Since $M(K)$ is orientable, $w_{2}(M(K))=0$, the equivariant intersection pairing on $\pi_{2}(M(K))$ is trivial and $\pi$ satisfies properties $W-A A, \pi$ determines $M(K)$ up to $s$-cobordism, by the main result of [9]. More precisely, if $K_{0}$ and $K_{1}$ each have group $\pi$ and their weight orbits correspond under an isomorphism $\theta: \pi K_{0} \cong \pi K_{1}$ then there is a (5-dimensional) $s$-cobordism $W$ from $M\left(K_{0}\right)$ to $M\left(K_{1}\right)$ such that $\pi_{1}\left(j_{0}\right)=\pi_{1}\left(j_{1}\right) \theta$, where $j_{i}: M\left(K_{i}\right) \rightarrow W$ is the natural inclusion, for $i=0,1$. The weight orbit of a meridian determines a properly embedded annulus in $W$ with boundary components meridians for $K_{0}$ and $K_{1}$. Excising an open product neighbourhood of this annulus gives a relative $s$ cobordism between the exteriors, and hence an $s$-concordance between the knots, modulo reflections and the Gluck ambiguity.

Beyond this, there is some reason to hope that all 2-knots with groups of deficiency 1 should be so determined. The exact state of affairs is contingent upon the outcome of several well-known conjectures. Let $\mathcal{D} \mathcal{W}$ be the class of groups with deficiency 1 and weight 1 . Kervaire showed that every group in $\mathcal{D W}$ is the group of a smooth knot in a smooth homotopy 4-sphere [18. He first constructs a knot manifold as the boundary of a 5-manifold obtained from $D^{5}$ by attaching 1 - and 2-handles, and so the groups are realized by homotopy ribbon knots.

If the Andrews-Curtis Conjecture is true then groups in $\mathcal{D} \mathcal{W}$ have Wirtinger presentations of deficiency 1 , and so are groups of ribbon 2-knots [29]. (In particular, these are smooth knots in $S^{4}$.) If the Whitehead Conjecture is true then g.d. $\pi \leq 2$, for all $\pi \in \mathcal{D} \mathcal{W}$. (If $\pi \in \mathcal{D W}$ then g.d.G $=2 \Leftrightarrow c . d . G=2$, by Theorem 2.8 of [11].) If the Fibred Isomorphism Conjecture holds for groups of cohomological dimension 2 then (ii) holds for such groups.

A conjecture which has perhaps been less studied has implications for characterizing 1-knot groups, and thus for recognizing the Artin spins of 1 -knots. If $\pi$ is a nontrivial 1 -knot group and $T \cong \mathbb{Z}^{2}$ is the 
subgroup generated by a meridian-longitude pair $(\mu, \lambda)$ then $(\pi, T)$ is a $P D_{3}$-pair. (When the knot is prime the subgroup $T$ is unique up to conjugacy.) If finitely presentable $P D_{3}$-groups are fundamental groups of aspherical closed 3-manifolds then the converse holds: every $P D_{3^{-}}$ pair $(\pi, T)$ in which $T \cong \mathbb{Z}^{2}$ contains a normal generator is the group of a 1-knot.

This is known in the fibred case. The following result is essentially Theorem 5 of [25], which is in turn a reformulation of Theorem 9.2.3 of [22], taking into account first the determination of $P D_{2}$-groups and pairs, and more recently Perelman's proof of the Poincaré Conjecture.

Theorem 6. Let $\pi$ be a group with deficiency 1 and weight 1, and suppose that $\pi \neq \mathbb{Z}$. Then the following are equivalent:

(1) $\pi$ is the group of a fibred 1-knot;

(2) $\pi$ has a subgroup $\Delta \cong \mathbb{Z}^{2}$ which contains a normal generator and such that $(\pi, \Delta)$ is a $P D_{3}$-pair, and $\pi^{\prime}$ is finitely generated;

(3) $\pi$ has a subgroup $\Delta=\langle\mu, \lambda\rangle \cong \mathbb{Z}^{2}$, where $\mu$ is a normal generator and $\lambda \in \pi^{\prime \prime}$, and such that $\left(\pi^{\prime},\langle\lambda\rangle\right)$ is a $P D_{2}$-pair.

Proof. If $\pi \cong \pi k$, where $k$ is a nontrivial 1-knot, then $X(k)$ is aspherical, $\partial X(k)$ is an incompressible torus, and $\pi$ is normally generated by a meridian on $\partial X(k)$. Hence $\Delta=\pi_{1}(\partial X(k)) \cong \mathbb{Z}^{2}$ contains a normal generator and $(\pi, \Delta)$ is a $P D_{3}$-pair. If $X(k)$ fibres over $S^{1}$ with fibre $F$ then $\pi^{\prime} \cong \pi_{1}(F)$ is finitely generated. Thus (1) implies (2).

If (2) holds then $\pi / \pi^{\prime} \cong \mathbb{Z}$, and so $T$ has a basis $\mu, \lambda$, where $\mu$ is a normal generator and $\lambda$ generates $T \cap \pi^{\prime} \cong \mathbb{Z}$. In fact, $\lambda$ is in $\pi^{\prime \prime}$, since $\mu$ commutes with $\lambda$ and $\pi / \pi^{\prime} \cong \mathbb{Z}$. Since $\operatorname{def}(\pi)=1$ and $\pi^{\prime}$ is finitely generated, $\pi^{\prime}$ is free, and so is $F P$. Hence $\left(\pi^{\prime},\langle\lambda\rangle\right)$ is a $P D_{2}$-pair, by a Shapiro's Lemma argument, as in Theorem 1.19 of [11. Thus (2) implies (3).

If $(3)$ holds then $\left(\pi^{\prime},\langle\lambda\rangle\right)$ is the fundamental group pair of a surface $S$ with boundary $\partial S=S^{1}$ [4]. The group $\pi$ is a semidirect product $\pi \cong \pi^{\prime} \rtimes_{\theta} \mathbb{Z}$, where the monodromy $\theta$ fixes $\lambda$. Hence it is realized by a self-homeomorphism $f$ of $S$, by Nielsen's Theorem. (See Theorem 5.7 .1 of [30].) We may adjoin a solid torus to the mapping torus $M(f)$ to get a homotopy 3-sphere containing a knot with exterior $M(f)$. Thus $\pi$ is the group of a fibred 1-knot, by the validity of the Poincaré Conjecture.

Corollary 6.1. A nontrivial 2-knot with group $\pi$ and meridian $\mu$ is s-concordant to the Artin spin of a fibred 1-knot if and only if $\pi^{\prime}$ is finitely generated, $C_{\pi}(\mu) \cong \mathbb{Z}^{2}$ and $\left(\pi, C_{\pi}(\mu)\right)$ is a $P D_{3}$-pair. 
Proof. This follows immediately from Theorems 5 and 6, since Artin spins of 1-knots are reflexive [7].

\section{REFERENCES}

[1] Aravinda, C.S., Farrell, F.T. and Roushon, S.K. Surgery groups of knot and link complements, Bull. London Math. Soc. 29 (1997), 400-406.

[2] Bieri, R. Homological Dimension of Discrete Groups, Queen Mary College Lecture Notes in Mathematics, London (1976).

[3] Cappell, S.E. Mayer-Vietoris sequences in Hermitean $K$-theory, in Hermitean $K$-Theory and Geometric Applications (edited by H.Bass), Lecture Notes in Mathematics 343, Springer-Verlag, Berlin - Heidelberg - New York (1973), $478-512$.

[4] Eckmann, B. and Müller, H. Poincaré duality groups of dimension two, Comment. Math. Helvetici 55 (1980, 510-520.

[5] Fox, R.H. A quick trip through knot theory, in Topology of 3-Manifolds and Related Topics (edited by M.K.Fort, Jr), Prentice-Hall, Englewood Cliffs, N.J.(1962), 120-167.

[6] Geoghegan, R. Topological Methods in Group Theory, Graduate Texts in Mathematics 243, Springer-Verlag, Berlin - Heidelberg - New York (2008).

[7] Gluck, H. The embedding of two-spheres in the four-sphere, Trans. Amer. Math. Soc. 104 (1962), 308-333.

[8] Gompf, R. More Cappell-Shaneson spheres are standard, Alg. Geom. Top 10 (2010), 1665-1681.

[9] Hambleton, I., Kreck, M. and Teichner, P. Topological 4-manifolds with geometrically two-dimensional fundamental groups, J. Topol. Anal. 1(2009), 123151.

[10] Hausmann, J.-C. and Kervaire, M. Sur le centre des groupes de noeuds multidimensionelles, C.R. Acad. Sci. Paris 287 (1978), 699-702.

[11] Hillman, J. A. Four-Manifolds, Geometries and Knots, Geometry and Topology Monographs 5, Geometry and Topology Publications (2002). (Revisions 2007 and 2014).

[12] Hillman, J.A. Strongly minimal $P D_{4}$-complexes, Top. Appl. 156 (2009), 15651577.

[13] Hillman, J.A. $P D_{4}$-complexes and 2-dimensional duality groups, arXiv: 1303.5486 v7 [math.GT]

[14] Hillman, J.A. and Howie, J. Seifert fibred knot manifolds, J. Knot Theory Ramif. 22 (2013), 1350082.

[15] Hillman, J.A. and Linnell, P.A. Elementary amenable groups of finite Hirsch length are locally finite by virtually solvable, J. Austral. Math. Soc. 52 (1992), $237-241$.

[16] Jensen, C.U. Les foncteurs dérivées de lim et ses applications a la théorie des modules, Lecture Notes in Mathematics 254, Springer-Verlag, Berlin Heidelberg - New York (1972).

[17] Kanenobu,T. Groups of higher dimensional satellite knots, J. Pure Appl. Alg. 28 (1983), 179-188.

[18] Kervaire, M.A. Les noeuds de dimensions supérieures, Bull. Soc. Math. France 93 (1965), 225-271. 
[19] Klyachko, A. Funny property of sphere and equations over groups, Comm. Alg. 21 (1993), 2555-2575.

[20] Kropholler, P.H. Soluble groups of type $(F P)_{\infty}$ have finite torsion-free rank, Bull. London Math. Soc. 25 (1993), 558-566.

[21] Lück, W. $L^{2}$-Invariants: Theory and Applications to Geometry and K-Theory, Ergebnisse der Mathematik und ihrer Grenzgebiete 3 Folge, Bd. 44, SpringerVerlag, Berlin - Heidelberg - New York (2002).

[22] Neuwirth, L.P. Knot Groups, Ann. Math. Studies 56, Princeton University Press, Princeton, N.J. (1965).

[23] Robinson, D.J.S. A Course in the Theory of Groups, Graduate Texts in Mathematics 80, Springer-Verlag, Berlin - Heidelberg - New York (1982).

[24] Strebel, R. A remark on subgroups of infinite index in Poincaré duality groups, Comment. Math. Helvetici 52 (1977), 317-324.

[25] Thomas, C.B. Splitting theorems for certain $P D^{3}$-groups, Math. Z. 186 (1984), 201-209.

[26] Yanagawa, T. On ribbon 2-knots: the 3-manifold bounded by the 2-knot, Osaka J. Math. 6 (1969), 447-464.

[27] Yoshikawa, K. On 2-knot groups with the finite commutator subgroups, Math. Seminar Notes Kobe University 8 (1980), 321-330.

[28] Yoshikawa, K. On a 2-knot group with nontrivial centre, Bull. Austral.Math. Soc. 25 (1982), 321-326.

[29] Yoshikawa, K. A note on Levine's condition for knot groups, Math. Sem. Notes Kobe University 10 (1982), 633-636.

[30] Zieschang, H., Vogt, E. and Coldewey, H.-D. Surfaces and Planar Discontinuous Groups, Lecture Notes in Mathematics 835, Springer-Verlag, Berlin Heidelberg - New York (1980).

School of Mathematics and Statistics, University of Sydney, NSW 2006, Australia

E-mail address: jonathan.hillman@sydney.edu.au 